\title{
OctoMag: An Electromagnetic System for 5-DOF Wireless Micromanipulation
}

\author{
B. E. Kratochvil, M. P. Kummer, J. J. Abbott, R. Borer, O. Ergeneman, and Bradley J. Nelson
}

\begin{abstract}
We demonstrate five-degree-of-freedom (5-DOF) wireless magnetic control of a fully untethered microrobot with a magnetic steering system we call OctoMag. Although only occupying a single hemisphere, this system is capable of isotropically applying forces on the order of 1-40 $\mu \mathrm{N}$ with unrestricted control of the 2 orienting DOF. These capabilities are enabled through the use of soft-magnetic-cores which provide an increase of approximately $20 \times$ that of air cores in magnetic-field strength, but comes at the cost of more complicated interactions between coils. We propose a modeling mechanism that assumes the field contributions of the individual currents superimpose linearly when using cores with large linear regions and negligible hysteresis. When designing the system, the locations and quantity of electromagnets were optimized with regards to the force generation in the worstcase direction predicted by the model. The resultant system is capable of both open and closed-loop operation over a workspace of $4 \mathrm{~cm}^{3}$. OctoMag was primarily designed for the control of intraocular microrobots for delicate retinal procedures, but also has potential uses in other medical applications or micromanipulation under an optical microscope.
\end{abstract}

\section{INTRODUCTION}

One approach to the wireless control of microrobots is through externally applied magnetic fields. These untethered devices can navigate in bodily fluids to enable a number of new minimally invasive therapeutic and diagnostic medical procedures. We are particularly interested in intraocular microrobots, which have the potential to be used in ophthalmic procedures such as drug delivery and remote sensing [1]. One particularly difficult procedure for vitreoretinal surgeons to perform is retinal-vein cannulation-the injection of a clotbusting enzyme into a tiny vein - which is at the limits of human capabilities [2]. A few groups have proposed robotassisted solutions for vitreoretinal surgery to attenuate the surgeon's hand tremor [3], [4]. In these proposed robotic solutions, the invasiveness of the procedure is not necessarily reduced, and the delicate retina is still at risk from a tool that is capable of inflicting irreparable damage.

In an effort to enable less invasive and safer retinal surgery, as well as providing an increased level of dexterity desired by clinicians, we embarked on the design of a system for magnetic manipulation of a fully untethered dexterous microrobotic device inside the eye. As opposed to manual surgery and existing robots, which are fundamentally position controlled, a magnetic device is fundamentally force controlled, with localization required for closed-loop

This work is supported by the NCCR Co-Me of the Swiss National Science Foundation. The authors are with the Institute of Robotics and Intelligent Systems, ETH Zurich, 8092 Zurich, Switzerland. e-mail: bnelson@ethz.ch. J. J. Abbott is now with the Department of Mechanical Engineering, University of Utah, Salt Lake City, UT, 84112 USA position control. This makes a magnetic tool a safer device for interacting with the retina: we can impose limits on the system to make irreparable retinal damage impossible, even in the event of patient movement or system failure. Ophthalmic procedures are also unique among minimally invasive medical procedures in that they provide a direct line of sight for visual feedback, making closed-loop position control of intraocular microrobots possible.

\section{EXPERIMENTAL RESULTS}

The prototype system is shown in Fig. 1, and consists of eight stationary electromagnets with ferromagnetic cores. Whereas magnetic manipulation has typically relied on orthogonal electromagnetic arrangements generating uniform fields, which are simple in terms of modeling and control, OctoMag's unprecedented level of wireless control is due to its utilization of complex nonuniform magnetic fields. In its current configuration the OctoMag is capable of creating field gradients up to $2 \mathrm{~T} / \mathrm{m}$ and orienting fields up to $50 \mathrm{mT}$ while steering the robot. A variety of devices is the millimeter and submillimeter range can be controlled as end effectors. We have thus far experimented with devices made from microassembled CoNi pieces, stainless steel tubing, and $\mathrm{NdFeB}$ permanent magnets.

Earnshaw's theorem tells us that there can be no stable static equilibria using ferromagnetism. That is, to maintain a stable position of the microrobot, we must use feedback control. Once we have implemented gravity compensation, we empirically find that the drift of the microrobot in a static field is quite slow. During open-loop control, a $500 \mu \mathrm{m}$ microrobot drifts with a velocity of roughly $50 \mu \mathrm{m} / \mathrm{s}$ at the

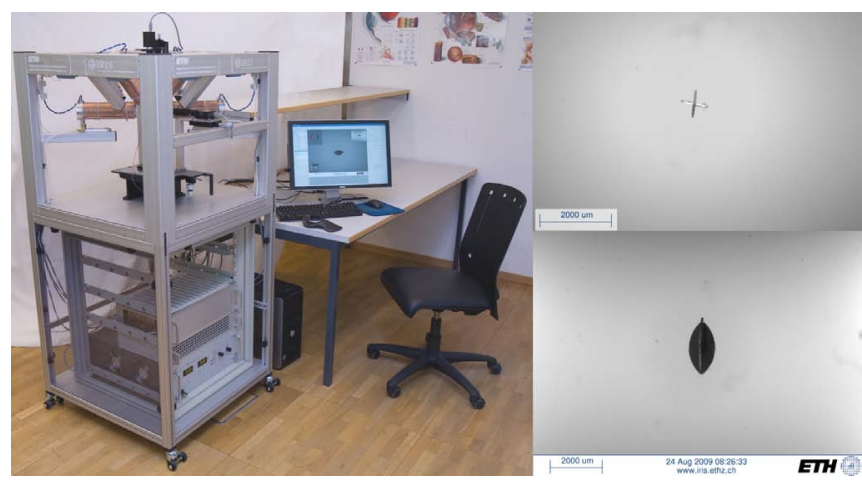

Fig. 1. The OctoMag prototype contains eight 210-mm-long by $62-\mathrm{mm}$ diameter electromagnets. The gap between two opposing electromagnets on the lower set is $130 \mathrm{~mm}$. The insets are the top and side-camera views, and show a 2-mm-long microrobot of the type described in [5]. 


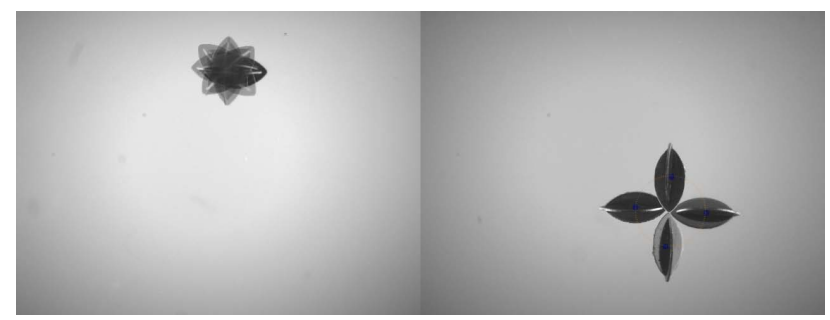

Fig. 2. Demonstration of rotation control. Both time-lapse image sequences show a single 2-mm-long microrobot in the $z=0$ plane (viewed from above). The left image sequence demonstrates rotation of the microrobot in place at an arbitrary location in space by relying on pure open-loop control. The right image sequence demonstrates rotation of the microrobot about a remote center at an arbitrary location using closed-loop control.

center of the workspace and $140 \mu \mathrm{m} / \mathrm{s}$ at the extremity of the workspace. We find that the human operator can regulate the position quite well using only visual feedback (Fig. 2), although not with the level of precision as when using the computer-vision tracker for closed-loop control (Fig. 3).

A chick choriallantoic membrane (CAM) has been demonstrated to be a viable model tissue for surgical retinal research and simulation [6]. Figure 4 shows a permanent magnetic device equipped with a needle tip being used to puncture a blood vessel. This demonstrates that an untethered magnetic agent is able to be manipulated with enough dexterity and force to potentially deliver enzymes or other clot-busting agents to the delicate structures of the eye.

Although OctoMag was designed for the control of intraocular microrobots for minimally invasive retinal therapy and diagnosis, it additionally has great potential for use as a wireless micromanipulation system under a light microscope.

\section{REFERENCES}

[1] O. Ergeneman, G. Dogangil, M. P. Kummer, J. J. Abbott, M. K. Nazeeruddin, and B. J. Nelson, "A magnetically controlled wireless optical oxygen sensor for intraocular measurements," IEEE Sensors J., vol. 8, no. 1, pp. 29-37, 2008.

[2] S. P. N. Singh and C. N. Riviere, "Physiological tremor amplitude during retinal microsurgery," in IEEE Northeast Bioengineering Conf., 2002, pp. 171-172.

[3] B. Mitchell, J. Koo, I. Iorcachita, P. Kazanzides, A. Kapoor, J. Handa, G. Hager, and R. Taylor, "Development and application of a new steadyhand manipulator for retinal surgery," in Proc. IEEE Int. Conf. Robotics and Automation, 2007, pp. 623-629.

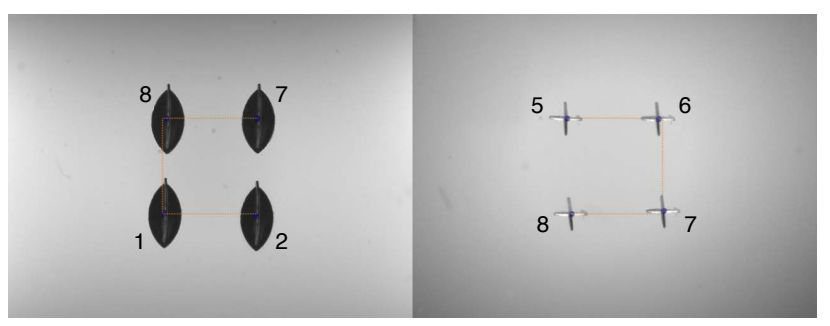

(a)

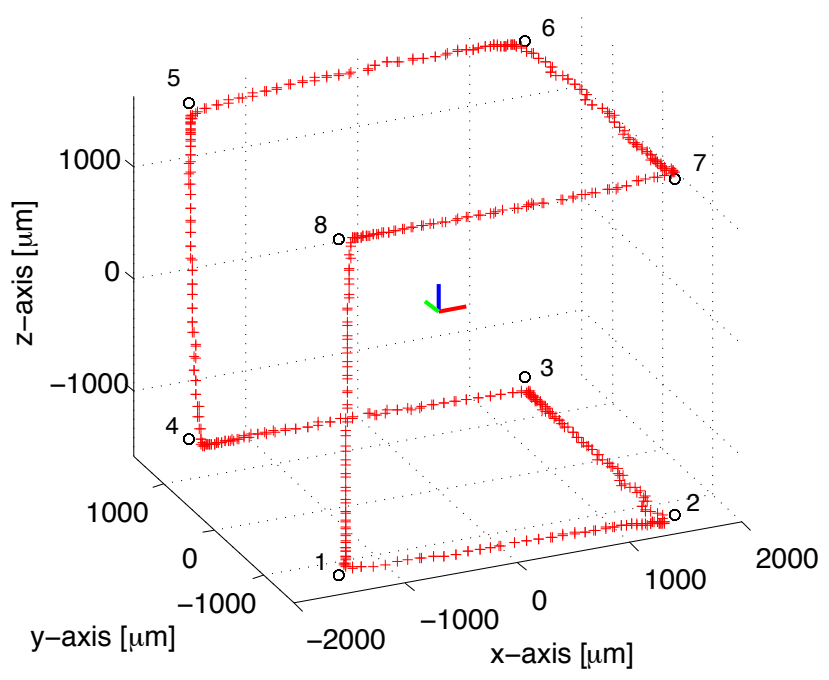

(b)

Fig. 3. Demonstration of automated position control. (a) show a 2-mmlong microrobot aligned with the $\mathrm{Z}$ axis (vertical). The left composite image demonstrates movement in a plane offset by $-1.5 \mathrm{~mm}$ off the $\mathrm{X}-\mathrm{Z}$ plane (viewed from the side). The right image sequence demonstrates movement in a plane offset $1.5 \mathrm{~mm}$ off the $\mathrm{X}-\mathrm{Y}$ plane (viewed from above). The microrobot was moved along the edges of a cube as displayed in the isometric graph (b). $\circ$ indicate waypoints. + indicate tracker data.

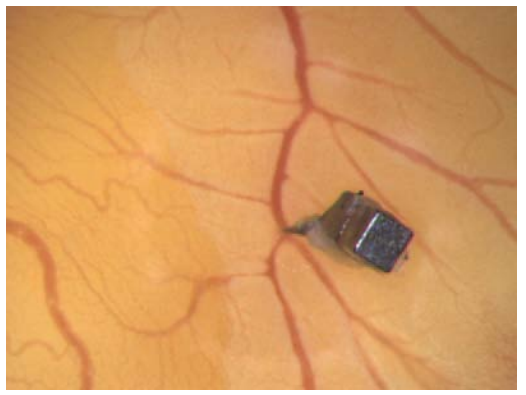

(a)

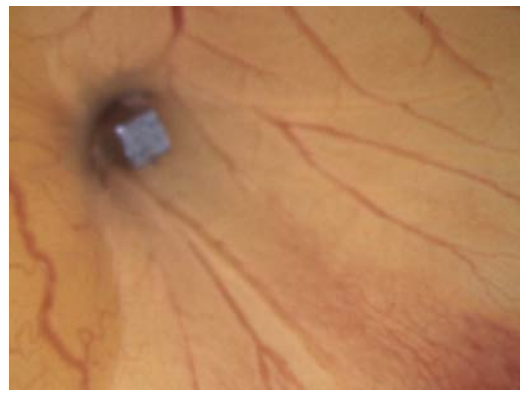

(b)

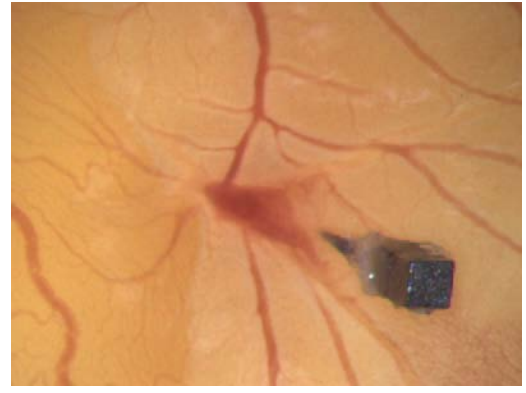

(c)

Fig. 4. The OctoMag controlling a 2.5-mm-long NdFeB agent with a needle tip to puncture vasculature on a CAM. 